\title{
Capacity Improvement in CDMA and FDMA Cellular Mobile Communication Systems Using Adaptive Antennas
}

\author{
Anand S. Sawant and Dinesh K. Anvekar* \\ Graduate Student Assistant Professor \\ Department of Electrical Communication Engineering \\ Indian Institue of Science, Bangalore 560012 India \\ E-mail: sawant@protocol.ece.iisc.ernet.in, dka@ece.iisc.emet.in
}

\begin{abstract}
A simulation study of the possibility of capacity improvement in a multi-cell CDMA and FDMA cellular systems by using adaptive antennas at the base stations is presented. Phased arrays that form steerable, fixed-pattern, narrow beams towards the users, have been assumed to approximate the performance of the adaptive antennas in a multipath-free environment. Simulation results for BER improvements in CDMA systems by assuming only distance dependent path-loss are given. Further improvements possible by considering voice activity factor are also presented. Then, simulation results for improvemants in the outage probability in CDMA and FDMA systems and degradation caused by power control errors assuming distance dependent path-loss and log-normal shadowing are presented.
\end{abstract}

\section{INTRODUCTION}

In the last few years, the demand for mobile communication services has increased tremendously. However, there is no proportionate increase in the spectrum allocated. As a result, there is an urgent need for new techniques to improve spectrum utilization by maximizing the number of users with the same available spectrum. One promising technique is to use antenna arrays at the Base Station (BS) for spatial filtering of signals. Spatial filtering reduces the amount of co-channel interference, and hence improves Carrier-to-Interference Ratio (CIR). This gain in CIR can be utilized for increasing the number of users per cell in CDMA and the reuse ratio in FDMA, and thus, improvements in system capacity can be achieved.

In this paper, we study the reverse link performance in CDMA and FDMA systems by employing adaptive antennas at the BSs for spatial filtering. The goal is to have a rough estimate of capacity improvements in interference limited CDMA and FDMA systems. Throughout this paper we assume phased arrays at the BSs, which form fixed pattern beams with adjustable boresights (i.e., steerable beams), to approximate the performance of adaptive antennas as proposed in [1]. We assume that the hardware and the adaptive algorithms for implementing the phased arrays can be realized. Further, we assume multipath-free environment.

The paper is organized as follows. In the next Section, we first present the use of the wedge-cell geometry and simulation method of [1] to obtain the bit error rate (BER) improvements in the reverse link of CDMA by using adaptive antennas at the BSs and also at the MSs. Then, we show through simulations that further improvements in capacity are possible by considering voice activity factor. Here, we assume only distance-dependent

* With IBM Solutions Résearch Center,India since October 1998. path loss, shadow-free environment, and perfect power control. Then, in section III, we extend the work presented in [1] by applying the phased array approximation of adaptive antennas to evaluate the improvements in uplink outage probability, $P_{\text {out }}$, in a CDMA system with hexagonal-cell geometry. Further, we obtain the degradation in $P_{\text {out }}$ caused by power control errors. A more accurate path loss, consisting of log-normal shadowing and a distance-dependent term, is used. Next, in Section IV, capacity improvements in FDMA systems are presented by using a procedure similar to that in Section III. Finally, concluding remarks are given in Section V.

\section{BER IMPROVEMENTS IN CDMA}

Here we use the wedge-cell geometry and simulation technique of [1]. The users are uniformly distributed throughout the region with an average of $\mathrm{K}$ users per cell. Each user is assigned to the nearest BS based on his geographical location. The path loss is assumed to be shadow-free and made of distance dependent component only. In the following presentation, we use the subscript triple $\langle i, j, k\rangle$ to denote that the concerned entity refers to the $i^{t h}$ user of the $j^{\text {th }}$ cell and the BS of the $k^{t h}$ cell. With this notation, the power received at BS $\mathrm{j}$ from the user $\mathrm{i}$ in that cell, $P_{R_{i, j, j}}$, is given by

$$
P_{R_{i, j, j}}=P_{T_{i, j}}\left(\frac{\lambda}{4 \pi d_{r e f}}\right)^{2}\left(\frac{d_{r e f}}{d_{i, j, j}}\right)^{n} G_{B S} G_{M S}
$$

where $P_{T_{i, j}}$ is the power transmitted by the user $\mathrm{i}$ of cell $\mathrm{j}, n$ is the propagation path loss exponent, $d_{r e f}$ is the close-in reference distance, and $G_{B S}$ and $G_{M S}$ are the gains of the BS and MS antennas, respectively. Since we assume perfect boresight alignment, both the gains are unity.

Next, we assume that each base station perfectly controls the transmitted power of each of its users so that each user in a cell will be received with the same power at that cell's BS. Thus, $P_{R_{i, j, j}}$ is constant, say, $P_{c}$. Then using the above equation, we can easily find $P_{T_{i, j}}$ in terms of $P_{c}$. The power received at the BS 0 (BS of the central cell), from the user $i$ of cell $j, P_{R_{i, j, 0}}$ is then given by

$$
P_{R_{i, j, 0}}=P_{T_{i, j}}\left(\frac{\lambda}{4 \pi d_{r e f}}\right)^{2}\left(\frac{d_{r e f}}{d_{i, j, 0}}\right)^{n} G_{B S} G_{M S}
$$

Substituting for $P_{T_{i, j}}$ we get

$$
P_{R_{i, j, 0}}=P_{c}\left(\frac{d_{i, j, j}}{d_{i, j, 0}}\right)^{n} G_{B S} G_{M S}
$$

Then, CIR for user $\mathrm{i}$ in cell $0, C I R_{i}$ is calculated as

$$
C I R_{i}=\frac{P_{i, 0,0}}{\sum_{p=0, p \neq i}^{K-1} P_{p, 0,0}+\sum_{m=1}^{8} \sum_{p=0}^{K-1} P_{p, m, 0}}
$$




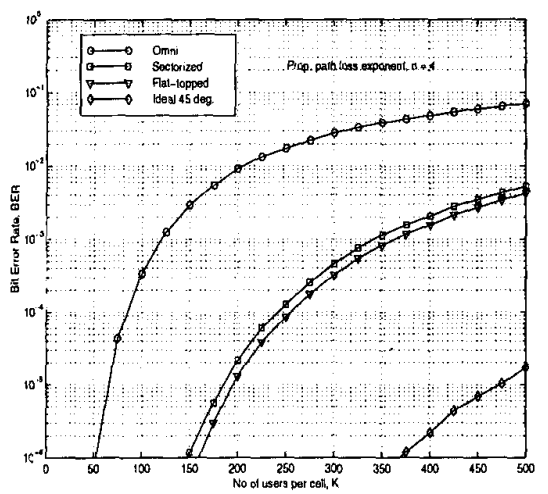

Fig. 1. BER obtaned by using 4 different BS antennas with omni antenna at the MS and $n=4_{k}$

where the first sum in the denominator is the in-cell interference while the second sum is the out-of cell interference. The main beam is directed towards each user in the central cell and CIR is calculated for that user. Then, by assuming that all users are asynchronous and by applying the Gaussian approximation, we get BER for user $i$ of cell 0 , as $B E R_{i}=Q\left(\sqrt{3 L * C I R_{i}}\right)$, where $L$ is the processing gain. The resultant BERs of all the users in the cell 0 are averaged to obtain an average BER for the cell.

Simulations have been carried out for $L=511$ and $n=2,3$, and 4. The artenna types used at the BS are (i) Omni, (ii) Sectorized (Fixecl sectors), (iii)Flat-topped $\left(30^{\circ}\right.$ wide uniform gain main beam and uniform side lobes with $6 \mathrm{~dB}$ lower gain), and ( $i v)$ Ideal $45^{\circ}$ (main beam width $=45^{\circ}$ ). Because of space constraints, the gain of the adaptive antenna at the MS will be considerably less. Therefore, we consider only omni and flat-topped $60^{\circ}$ antennas for the MS.

The results for $n=4$ and omni antenna at MS are shown in Fig, 1, and for flat-topped $60^{\circ}$ antenna at the MS in Fig. 2. The Ideal $45^{\circ}$ shows an improvement of better than three orders of magnitude over the omni antenna even for more than 300 users/cell. With the flat-topped $60^{\circ}$ at the MS, the improvement is less for ornni, sectorized and flat-topped antennas at the BS, while for Ideal $45^{\circ}$ antenna it is better than an order of magnitude even for more than 450 users/cell. Next, Fig. 3 shows further BER improvement for $n=4$ and omni antenna at MS by taking voice activity factor, $\alpha$, into account. It is to be observed that for $\alpha=0.4$ the increment in the number of users/cell for a given value of BER is approximately equal to $1 / \alpha$.

\section{OUTAGE PROBABILITY IMPROVEMENTS IN CDMA}

The outage probability, $P_{\text {out }}$, is defined as the probability that BER exceeds a certain threshold required for acceptable performance (e.g., $B E R<10^{-3}$ ) after decoding. As noted in [2], this is achieved with $E_{b} /\left(N_{0}+I_{0}\right) \geq 7 d B$. Assuming negligible background noise, this can be directly translated into the minimum required $\operatorname{CIR}, \gamma$, as $\gamma=\frac{\left(\frac{E_{b}}{N_{0}}\right)}{L}$ where $\mathrm{L}$ is the processing gain. Hence, the outage probability can also be defined as the probability that the CIR falls below $\gamma$ and is found from the CIR

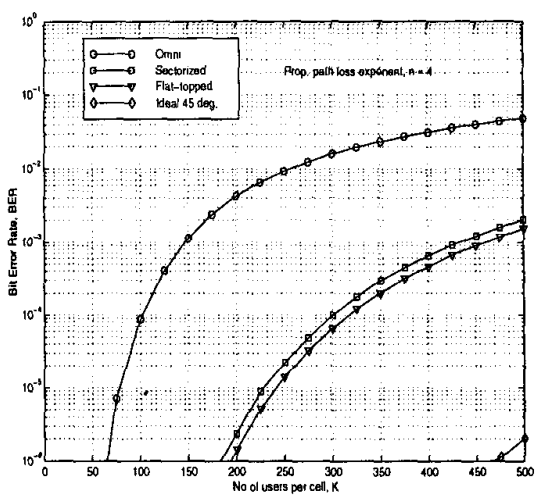

Fig. 2. BER obtained by using 4 different BS antennas with Flat-topped $60^{\circ}$ antenna at the MS and $n=4$

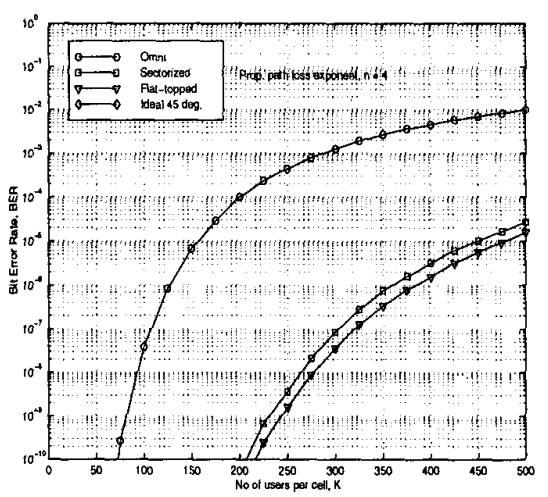

Fig. 3. BER obtained by using 4 different BS antennas with omni antenna at the MS after taking voice activity factor into account, $n=4$ and $\alpha=0.4$

distribution. Here we use the simulation technique of [3] to obtain the distribution for 5 different BS antenna types, viz., omni, sectorized, flat-topped, ideal $45^{\circ}$, and ideal $30^{\circ}$.

We consider a CDMA system consisting of a central cell and two rings of interfering. BSs with a hexagonal cell geometry. The users are uniformly distributed throughout the region with an average of $\mathrm{K}$ users/cell. The path loss consists of a distance-dependent term and shadowing. The shadowing is modelled by independent, log-normally distributed random variables with 0 mean and standard deviation $\sigma_{s}$. Then, the power received at the BS $\mathrm{j}$ from user $\mathrm{i}$ in cell $\mathrm{j}, P_{R_{i, j, j}}$, is given by

$$
P_{R_{i, j, j}}=P_{T_{i, j}}\left(\frac{\lambda}{4 \pi d_{r e f}}\right)^{2}\left(\frac{d_{r e f}}{d_{i, j, j}}\right)^{n} 10^{\frac{\zeta_{i, j, j}}{10}}
$$

where $G_{B S}$ and $G_{M S}$ are assumed to be 1 due to perfect boresight alignment and $\zeta_{i, j, j}$ is the log-normal shadowing along the path from the user $\mathrm{i}$ in cell $\mathrm{j}$ to $\mathrm{BS} \mathrm{j}$. Further, we assume perfect power control. Then, following the procedure outlined in Section II, we can obtain the power received at BS 0 from user $i$ in cell $j$ as

$$
P_{R_{i, j, 0}}=P_{c}\left(\frac{d_{i, j, j}}{d_{i, j, 0}}\right)^{n} 10^{\frac{\zeta_{i, j, 0}-\zeta_{i, j, j}}{10}} G_{B S} G_{M S}
$$

In the simulations, a large number of random deployments of users are generated. To simplify computation, we assign the users to BSs not on minimum path loss criterion but on minimum distance criterion. In [2], it has been proved that this leads to only 


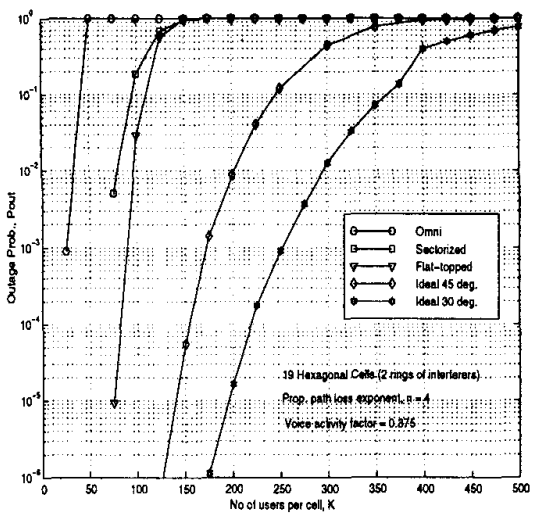

Fig. 4. $P_{\text {out }}$ in CDMA for different BS antenna types and with perfect power control

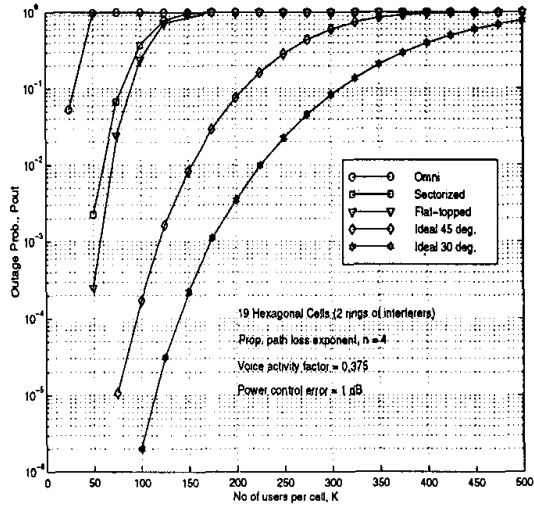

Fig. 5. $P_{\text {out }}$ in CDMA for different $\mathrm{BS}$ antenna types and with power control error; $\sigma_{p c e}=1 d B$

slightly higher values for outage probability. For each user deployment, CIR for the central cell user is found by using Equation (3). It is to be noted that the second sum runs for $m=1$ to 18 in this case. This is repeated 10000 times to get the CIR distribution. Fig. 4 shows the improvement in $P_{\text {out }}$ for $L=128, \alpha=0.375$, and $\sigma_{s}=8 d B$. It can be observed that, for $P_{\text {out }}=0.01$, the capacity increases 10 times, from about 28 users/cell for the omni antenna to about 300 users/cell for the ideal $30^{\circ}$ antenna.

Next, we incorporate the power control errors by scaling the MS transmit powers with independent, zero mean, log-normally distributed random variables having standard deviation $\sigma_{p c e}$. Fig. 5 shows the degradation of $P_{\text {out }}$ for $n=4, L=128, \alpha=0.375$, $\sigma_{s}=8 d B$, and $\sigma_{p c e}=1 d B$. It is to be noted that the capacity decreases by about $25 \%$ for $P_{\text {out }}=0.01$ for all antenna types.

\section{OUTAGE PROBABILITY IMPROVEMENTS IN FDMA}

Here we consider an FDMA system consisting of a central cell and one ring of interfering BSs with a hexagonal cell geometry. The users are uniformly distributed within each cell. The path loss consists of a distance-dependent term and shadowing. We assume perfect boresight alignment and perfect power control. Thus, this case is similar to the CDMA system considered in Section III with the exception that there is no in-cell interference, and there is only

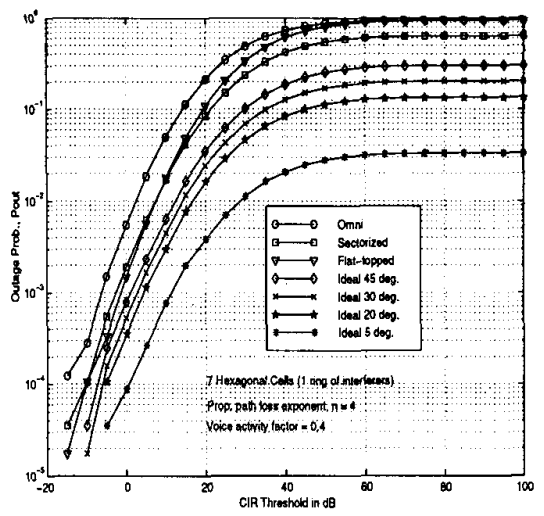

Fig. 6. Pout in FDMA for different BS antenna types and with perfect power control

one interferer in the cells of interfering BSs. Using a procedure similar to that used in Section III for CDMA, we can get the CIR distribution. Fig. 6 shows $P_{\text {out }}$ for omni, sectorized, flat-topped, ideal $45^{\circ}$, ideal $30^{\circ}$, ideal $20^{\circ}$, and ideal $5^{\circ}$ antennas obtained with reuse ratio $=7, n=4, \alpha=0.375, \sigma_{s}=8 d B$, and $\sigma_{p c e}=0 d B$. From the figure it is clear that, for $P_{\text {out }}=0.01$, the ideal $30^{\circ}$ anterna has a CIR threshold that is about $20 \mathrm{~dB}$ higher than that for omni antenna. Therefore, reuse ratio can be reduced to match this improvement in CIR, and hence, the capacity of the system can be increased.

\section{CONCLUSION}

In this paper, we have shown that the use of directional antennas at the BS alone leads to considerable increase in the capacity of CDMA and FDMA systems. Further, we have shown that these increments in capacity can be estimated by using phased arrays, that form steerable; fixed pattern beams, to approximate the performance of an adaptive antenna in a multipath-free environment. This has been demonstrated by first obtaining BER improvements in CDMA, and then studying the effect of considering voice activity factor on BER. Next, we have shown improvements in outage probabilities in CDMA and FDMA systems and studied the effect of power control errors. A line of further research work is to carry out a similar study by considering a multi-path propagation environment.

\section{REFERENCES}

[1] J. C. Liberti, Jr., and T. S. Rappaport, "Analytical Results for Capacity Improvements in CDMA", IEEE Trans. Veh. Technol., vol. 43, no. 3, pp. 680-690, Aug. 1994.

[2] K. S. Gilhousen, I. M. Jacobs, R. Padovani, A J. Viterbi, L A. Weaver, Jr., and C. E. Wheatley, "On the Capacity of Cellular CDMA System", IEEE Trans. Veh. Technol., vol. 40, no. 2, pp. 303-312, May 1991.

[3] B. Gudmundson, J. Skold, and J. K. Ugland, "A comparison of CDMA and TDMA systems", Proc. of the 42nd VTS Conference, May 10-13, Denver, Colorado, vol. 2, pp. 732 735 . 